\title{
Visualization of Cytoskeletal Elements in the Transmission X-ray Microscope
}

\author{
Dag Scherfeld, Gerd Schneider, Peter Guttmann, and Mary Osborn* \\ Institute of X-ray Physics, University of Goettingen, Geiststrasse 11, 37073 Goettingen, Germany; and *Department of Biochemistry, \\ Max Planck Institute for Biophysical Chemistry, Am Fassberg 11, D-37077 Goettingen, Germany
}

Received April 15, 1998, and in revised form J uly 8, 1998

Transmission X-ray microscopy has been used to study the arrangement of cytoskeletal filaments in interphase PtK2 cells. Extraction of the soluble proteins and of some organelles with the nonionic detergent Triton X-100 was important in obtaining sufficient image contrast between the insolublecytoskeletal filaments and the surrounding cytoplasm. If this step is not performed cytoskeletal filaments are not visualized and transmission X-ray micrographs of the cytoplasm instead show predominantly membrane-bound organelles such as vesicles and the endoplasmic reticulum. Transmission X-ray micrographs of the cytoskeletal filaments and endoplasmic reticulum in air-dried specimens, as well as in specimens examined in the wet state, can be directly compared with transmission electron micrographs of cytoskeletons prepared in the same way. The profiles seen with the two techniques are similar, although, currently, transmission X-ray micrographs have a limit of resolution of $\sim 50 \mathrm{~nm}$. Transmission $X$-ray micrographs appear to show some substructure in interphase nuclei in cells fixed either with glutaraldehyde or by cryofixation and examined in a hydrated condition. 1998 Academic Press

Key Words: actin; cytoskeleton; intermediate filaments; interphase cells; limit of resolution; X-ray microscopy.

\section{INTRODUCTION}

Transmission el ectron microscopy (TEM) and light microscopy are techniques that have made major contributions to our understanding of cellular and organelle morphology. In addition both techniques have allowed the description of the three filament systems in cells, i.e., microtubules, microfilaments, and intermediate filaments. In the electron microscope these filament systems can be distinguished by their differing diameters and by their different morphologies either in sectioned material or in whole mounts. I mmunofluor escence microscopy using anti- bodies specific for one of the filament systems, e.g., tubulin for microtubules (Osborn et al ., 1978; Osborn and Weber, 1977b; Weber et al., 1978), actin for microfilaments (Webster et al., 1978), or keratin for the epithelial type of intermediate filaments (Webster et al., 1978), allows the specific visualization of individual filament systems and is a quick and easy way to obtain an overview of the arrangements of these filaments in a variety of cell types. Control experiments have demonstrated a 1:1 correspondence between filaments visualized in the light and el ectron microscopes for both microtubules and actin filaments (Osborn et al., 1978; Webster et al., 1978).

Transmission X-ray microscopy (TXM) is a technique that is just beginning to be applied to biological material. This technique may bridge the gap between electron and light microscopy (Schmahl et al., 1980). TXM uses wavelengths in the range 0.3 to $5 \mathrm{~nm}$ so that with a modern transmission X-ray microscope, 10 times higher resolution can be obtained as with light microscopy (Kirz et al., 1995; Schmahl et al., 1995). In addition, whereas many cells are too thick to be visualized directly by TEM, specimens up to $10 \mu \mathrm{m}$ thick can be examined directly by TXM. Thus, TXM has the potential to visualize cell organelles or structures in whole cells at high resolution. In addition, micrographs can be made of cells in a near-normal environment, i.e., at normal atmospheric pressure while surrounded by liquid. TXM has the further advantage that carbon and oxygen show different photoelectric absorption when wavelengths between 2.4 and $4.5 \mathrm{~nm}$ are used (Wolter, 1952). Thus, TXM reveals a natural contrast between the organic compounds in the biological specimen and the surrounding water. TXM can also be used in the phase-contrast mode, resulting in better contrast especially for biological specimens (Schmahl et al., 1995). A disadvantage of both TXM and TEM, in comparison to light microscopy, is the need to stabilize specimens against radiation damage. This can be achieved, for instance, by fixing the 
material with glutaraldehyde, by air-drying, or by cryofixation. Radiation damage studies using soft $X$ rays have shown that glutaraldehyde-fixed cells in the wet state are structurally stable up to about $10^{6}$ Gy, which makes it possible to obtain a single image at about 50-nm resolution (Gilbert et al., 1992). Much higher doses up to $10^{10} \mathrm{~Gy}$ can be applied to cryofixed samples without inducing structural damage in the biological samples. This has been demonstrated by making successive images of the same area of frozen hydrated al ga (Schneider et al ., 1995a).

Intact mammalian cells fixed with glutaral dehyde have been examined by TXM (M ethe et al ., 1997) and by scanning TXM (Gilbert et al., 1992). In mitotic cells chromosomes as well as other elements of the mitotic apparatus were visualized by TXM. In interphase cells the nuclear membrane, chromatin, nucleolus, as well as mitochondria and vesides, were visualized in a single cell type - rat kangaroo PtK2 cells-but visualization of cytoplasmic filamentous structures has not been reported (Metheet al., 1997). The purpose of this study was to investigate the conditions under which such filament systems can be visualized by TXM. Here we show that detergent extraction (Lenk et al., 1977; Osborn and Weber, 1977a) allows the different cytoskeletal filament systems to be visualized in PtK 2 cells. Actin filaments as well as intermediate filaments are visualized at the limit of resolution of the transmission X-ray microscope, i.e., at $\sim 50 \mathrm{~nm}$.

\section{MATERIALS AND METHODS}

Preparation of support and cover foils. Both support and cover foils have been specially developed for X-ray microscopy and have a high transmission at a wavelength of $2.4 \mathrm{~nm}$, which is the wavelength used in this study (Niemann et al., 1994; Schneider, 1992). Both foils are built from stainless-steel rings (outer diameter, $25 \mathrm{~mm}$; inner diameter, $13 \mathrm{~mm}$ ) to which a $\sim 400-\mathrm{nm}$-thick polypropylene foil is glued with a special spray glue (Transotype, Wiesbaden, Germany). A soldering instrument is used to make a 3-mm-diameter hole in the center of each foil. Finally, a polyimide foil with a thickness of $\sim 125 \mathrm{~nm}$ is placed so that the hole is fully covered. The polypropylene foil is characterized by a high elasticity, whereas the polyimi de foil has low elasticity and is resistant to radiation damage. The polyimide foil is coated with $20-\mathrm{nm}$ layers of silicon and silicon dioxide to prevent distortion of the foil and to prevent its detachment. The silicon layer also helps reflect incident light so that the cells on the foil can be observed with an interference-contrast microscope while in the transmission X-ray microscope. The silicon dioxide layer is important for optimal cell growth. The cover foil is coated with a 50-nm layer of aluminum oxide. This is, unlikesilicon, transparent and allows the cells to be observed through the cover foil. The foils were sterilized by a short treatment ( $2-3 \mathrm{~min}$ ) in $70 \%$ ethanol. It is important that reagentgrade ethanol without additives is used for this purpose. If impure ethanol is used, bubbles developed during the subsequent incubation of the foils in medium. Foils can be used without ethanol sterilization if they have been prepared carefully and are stored appropriately. The foils can be stored in a closed container for at least 1 month before use.
Cells. Cells of the established rat kangaroo cell line PtK 2 were grown in Dulbecco's modified Eagle's medium with added glutamine, nonessential amino acids, pyruvate, and $10 \%$ fetal calf serum in an atmosphere containing $5 \% \mathrm{CO}_{2}$ (Webster et al., 1978). Support foils were preincubated overnight in the above medium without cells. Cells were trypsinized and diluted onto the foils in fresh medium. After $24 \mathrm{~h}$ they had attached and were halfconfluent.

Cytoskeleton preparations for TXM. All extraction and wash steps were performed in 5-cm-diameter plastic Petri dishes at room temperature according to the procedure of Webster et al. (1978). Support foils with attached cells were washed once in buffer A $\left(0.01 \mathrm{M}\right.$ Tris- $\mathrm{HCl}$, pH 7.6, $0.14 \mathrm{M} \mathrm{NaCl}, 0.005 \mathrm{M} \mathrm{MgCl}_{2}$, $4 \%$ polyethylene glycol 6000 ). They were then incubated for $1-3$ min in buffer A with $0.5 \%$ Triton X-100. They were washed twice for $2 \mathrm{~min}$ in buffer $A$ and then fixed in buffer $A$ with $1 \%$ glutaral dehyde for $10 \mathrm{~min}$. Unstained cytoskel etons were kept in buffer A with glutaraldehyde until they could be examined by TXM. Other samples were stained with uranyl acetate. Immediately after fixation in glutaraldehyde a small volume of a $1 \%$ aqueous solution of uranyl acetate was pipetted onto the grid so that it covered the cells. After 45-60 s excess uranyl acetate solution was removed carefully by applying filter paper to the drop. Some samples were washed twice more with distilled water. The samples were air-dried and stored at room temperature.

Wholecell preparations for TXM. Support foils with attached cells were removed from the medium, washed once in buffer $A$, and fixed in buffer A with $1 \%$ glutaraldehyde. They were kept in this buffer until they could be examined by TXM.

Cytoskeleton wholemount preparations for TEM. Cells were grown for $24 \mathrm{~h}$ on 100-mesh gold grids instead of on support foils. The grids were sandwiched between a $1.2 \%$ Formvar film and a $12-\mathrm{mm}$ glass coverslip. Cells were extracted for $3 \mathrm{~min}$ at room temperature in the same buffers used above for the TXM samples. Samples were then washed once with $0.3 \mathrm{M} \mathrm{KCl}$ and once with water and then stained with uranyl acetate (for further details, see Webster et al., 1978). Cells were examined in a Philips CM 12 transmission electron microscope and photographed directly.

X-ray microscope and digital image processing. All transmission X-ray micrographs were made on the transmission X-ray microscope of the X-ray physics facility of the University of Göttingen at the Berlin Electron Storage Ring for Synchrotron Radiation (BESSY). An X-ray phase-contrast objective with which it was possible to make both amplitude and phase-contrast micrographs by varying the object illumination was used. The theoretical resolution of the optical system was $48 \mathrm{~nm}$ when used at a wavelength of $2.4 \mathrm{~nm}$. The specimens were examined by TXM and the images were recorded with a cooled slow-scan CCD camera with a thinned, back illuminated chip (Photometrics, Munich, Germany) with a primary magnification of 1496 . The exposure time for the amplitude-contrast pictures was 2-5 s, and for the phase-contrast pictures, $15-60 \mathrm{~s}$. Thus, the micrographs were already in digital form. Since the object area that can be imaged is limited to $16 \times 16 \mu \mathrm{m}^{2}$, and a PtK 2 cell can be up to 50 $\mu \mathrm{m}$ long, multiple overlapping images have to be combined to obtain the image of a single PtK 2 cell. To do this the individual images were subjected to background correction with the help of morphological image processing. This was done by nonlinear filter processing. The image was scanned using a preprogrammed sensor to increase the contrast of the fine structure present in the image while reducing the nonlinear background caused by object illumination (for further details see Lehr, 1993; Boomgaard, 1992). This increased the contrast of the structures present in the images. Then the brightness and contrast of the individual images were varied until an optimal image was obtained. The individual images were then combined. In this process the images obtained 
from the CCD camera were enlarged by a factor of 3-10, giving an overall magnification of the specimens of 5000-15 000 .

\section{RESULTS}

\section{Specimen Chambers}

Almost the entire path taken by the ionizing radiation in the transmission X-ray microscope is in a vacuum. The vacuum is interrupted only at the position where the specimen is inserted, and thus the specimen is observed at atmospheric pressure (Niemann et al., 1994). The space into which the specimen is inserted has to be kept small because soft $X$ rays are strongly absorbed in air. This necessitates the devel opment of special specimen chambers.

The chamber for wet specimens is constructed from a support foil and a cover foil. Each foil is mounted on a $100-\mu$ m-thick metal ring. The two foils are held apart by a third metal ring $100 \mu \mathrm{m}$ thick (Fig. 1). I nitially the spacing between the two foils is $300 \mu \mathrm{m}$, and this space is filled with water. The thickness can be measured directly by differential interference-contrast microscopy. The thickness is reduced to $10 \mu \mathrm{m}$ using two syringes built into the central ring of the specimen holder, so that sufficient $X$ rays can be transmitted through the chamber.

For air-dried specimens only the support foil is used without the spacing ring and the cover foil. This is inserted directly into the transmission X-ray microscope.

\section{Observation of WholeCells}

It is striking that thus far no components of the cytoskel eton have been visualized in interphase cells viewed in the X-ray microscope (cf. Gilbert et al., 1992; Methe et al., 1997). This is illustrated in Fig. $2 \mathrm{a}$, which shows part of a rat kangaroo PtK 2 cell that was fixed with glutaraldehyde, then observed in the $X$-ray microscope and imaged as a wet specimen in the phase-contrast mode. While lipid-containing organelles such as mitochondria and parts of the endoplasmic reticulum are clearly seen, the three cytoskeletal filament systems, i.e., actin filaments, intermediate filaments, and microtubules, are not.
The nucleus and the nuclear lamina can be distinguished at the top right of the figure. Some substructure may be apparent in the nucleus. For comparison, Fig. 2 b shows a PtK 2 cell that has been snapfrozen in liquid ethane at $-173^{\circ} \mathrm{C}$ (cf. Schneider et al., 1995a). Mitochondria and other membranecontaining structures such as vesicles are present, but again cytoskeletal elements are not visible. Substructure within the nucleus is clearly apparent in the cryofixed specimen.

\section{Observation of Cytoskel etons}

Previous experiments on tissue culture cells have shown that treatment with detergents such as Triton X-100 both permeabilize the cell and extract much of the soluble proteins from the cell while leaving the cytoskeleton intact (Osborn and Weber, 1977a; Lenk et al., 1977; Webster et al., 1978; Fey et al., 1984). The extent of extraction depends on the concentration and type of detergent selected and on the temperature and time for which the extraction is performed.

A micrograph of a PtK 2 cell treated with the nonionic detergent Triton X-100, air-dried, stained with uranyl acetate, and viewed in the transmission $X$-ray microscope in the air-dried state is shown in Fig. 3. This micrograph shows a cytoplasmic area of a PtK 2 cell equivalent to the left-hand part of Fig. 2. Thick stress fibers, as well as thinner filaments, can be seen in the micrographs. The identical field has been photographed with amplitude contrast in Fig. $3 a$ and in the phase-contrast mode in Fig. 3b. Comparison of Figs $3 a$ and $3 b$ shows that the contrast is clearly better in the phase-contrast mode.

Figure 4 shows the results of varying the times of extraction and of observing the cytoskeleton in the dry or wet state. The specimens in Figs. $4 a$ and $4 b$ underwent $1 \mathrm{~min}$ of extraction, and these images are from regions showing predominantly strong stress fibers and thinner filaments. The specimens in Figs. $4 c$ and $4 d$ underwent $3 \mathrm{~min}$ of extraction, and the images are taken from a region of the cell closer to the nucleus and therefore contain elements of the

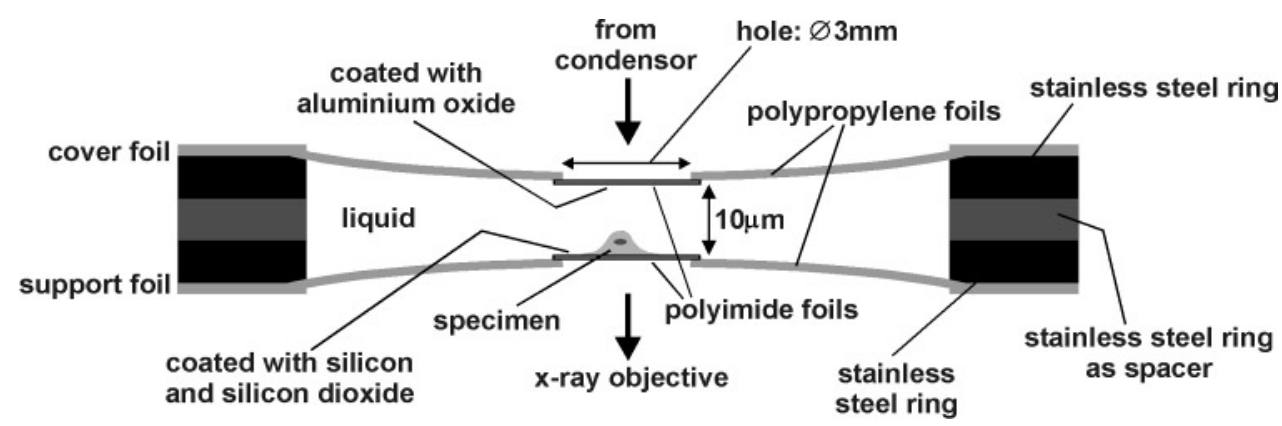

FIG. 1. Diagram showing the specimen chamber used for wet probes. 

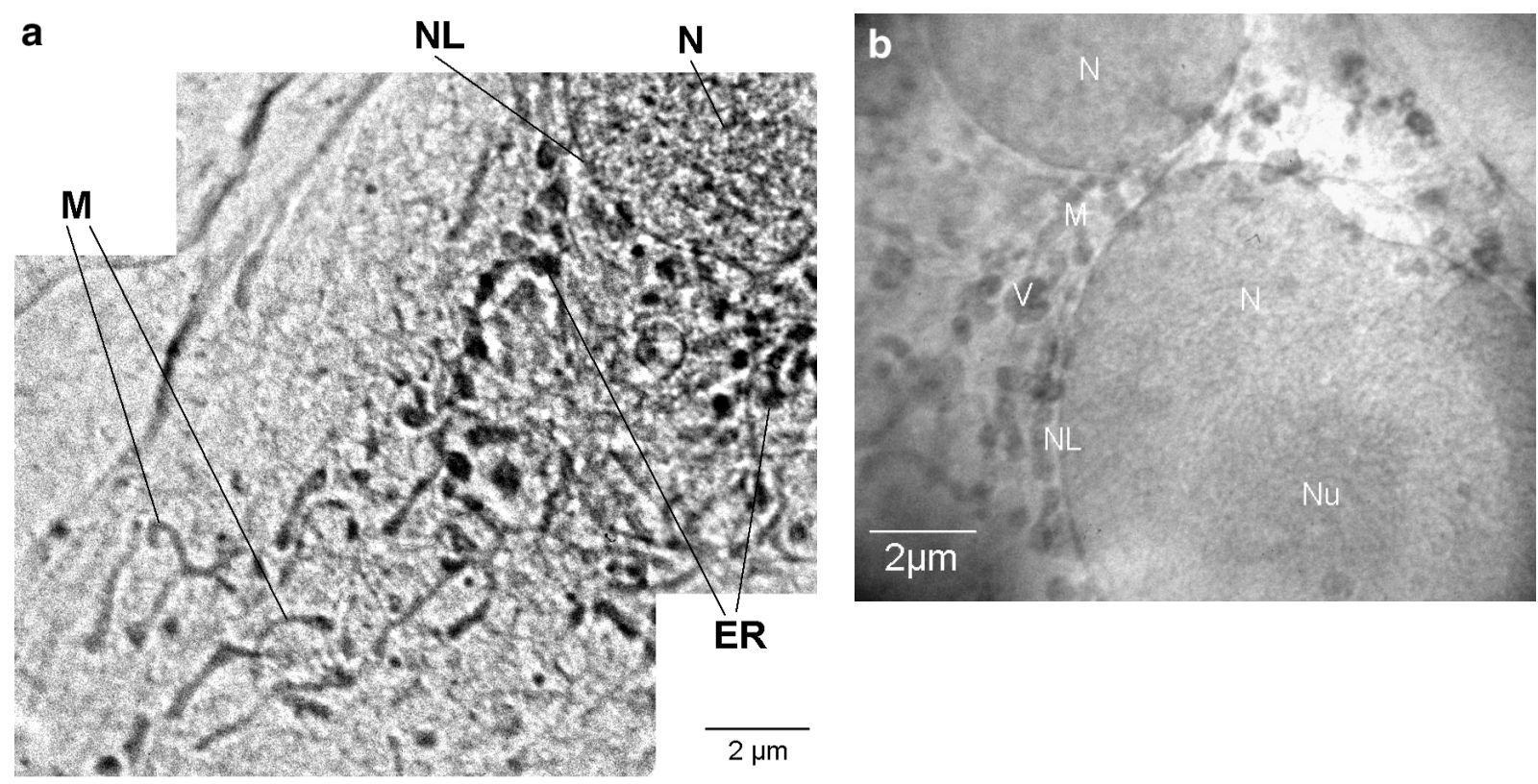

FIG. 2. (a) X-ray micrograph in the phase-contrast mode of an unextracted, unstained PtK 2 cell that has been fixed in glutaral dehyde and photographed as a wet probe. The nucleus $(\mathrm{N})$ and the nuclear lamina (NL) are visible at the top right. Cytoskeletal elements are not visualized but mitochondria (M) and parts of the endoplasmic reticulum (ER) can be seen. (b) X-ray micrograph of PtK2 cells fixed by plunging into liquid ethane, made in the cryo-transmission $\mathrm{X}$-ray microscope. Nucleus ( $\mathrm{N}$ ), nucleolus ( $\mathrm{Nu}$ ), nuclear lamina ( $\mathrm{NL}$ ), vesicles (V), and mitochondria (M) are visible.

endoplasmic reticulum in addition to filamentous structures. The images in Figs. $4 \mathrm{a}$ and $4 \mathrm{c}$ are of uranyl acetate-stained, dried specimens, while the images in Figs. $4 b$ and $4 d$ are of non-uranyl acetatestained, wet specimens. Comparison of Figs. $4 a-4 d$ shows that cytoskeletal elements, including both the thick bundles of stress fibers and the thinner filaments, are most clearly visualized in the probes extracted for the longer times. After 1 min extraction a better contrast is seen in the dry state (Fig. 4a) than in the wet state (Fig. 4b). After 3 min extraction stress fibers can be visualized in both the dry (Fig. 4c) and wet (Fig. 4d) states. However, the contrast is better in the dry than in the wet state. This is due not only to the water molecules covering the cytoskeletal structures in the wet state, which reduces the
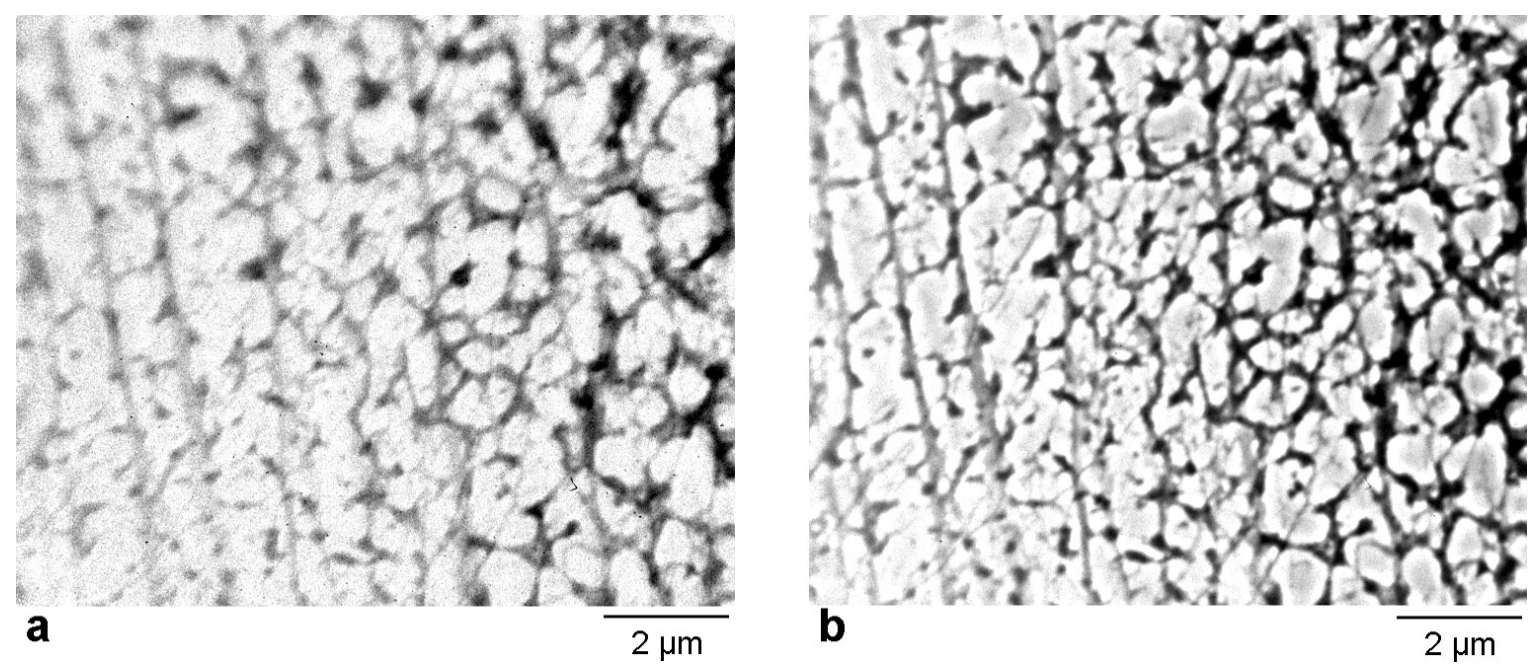

FIG. 3. These X-ray micrographs show the same cytoplasmic area from the same PtK 2 cytoskeleton. The specimen was prepared by extracting the cells with detergent, air-drying, labeling with uranyl acetate, and observing in the air-dried state. (a) Made with amplitude contrast. (b) Same area as in (a) imaged in the phase-contrast mode. Note, in contrast to Fig. 2, that thick stress fibers, as well as smaller filaments, are visible in both (a) and (b). Note the better contrast in (b). 

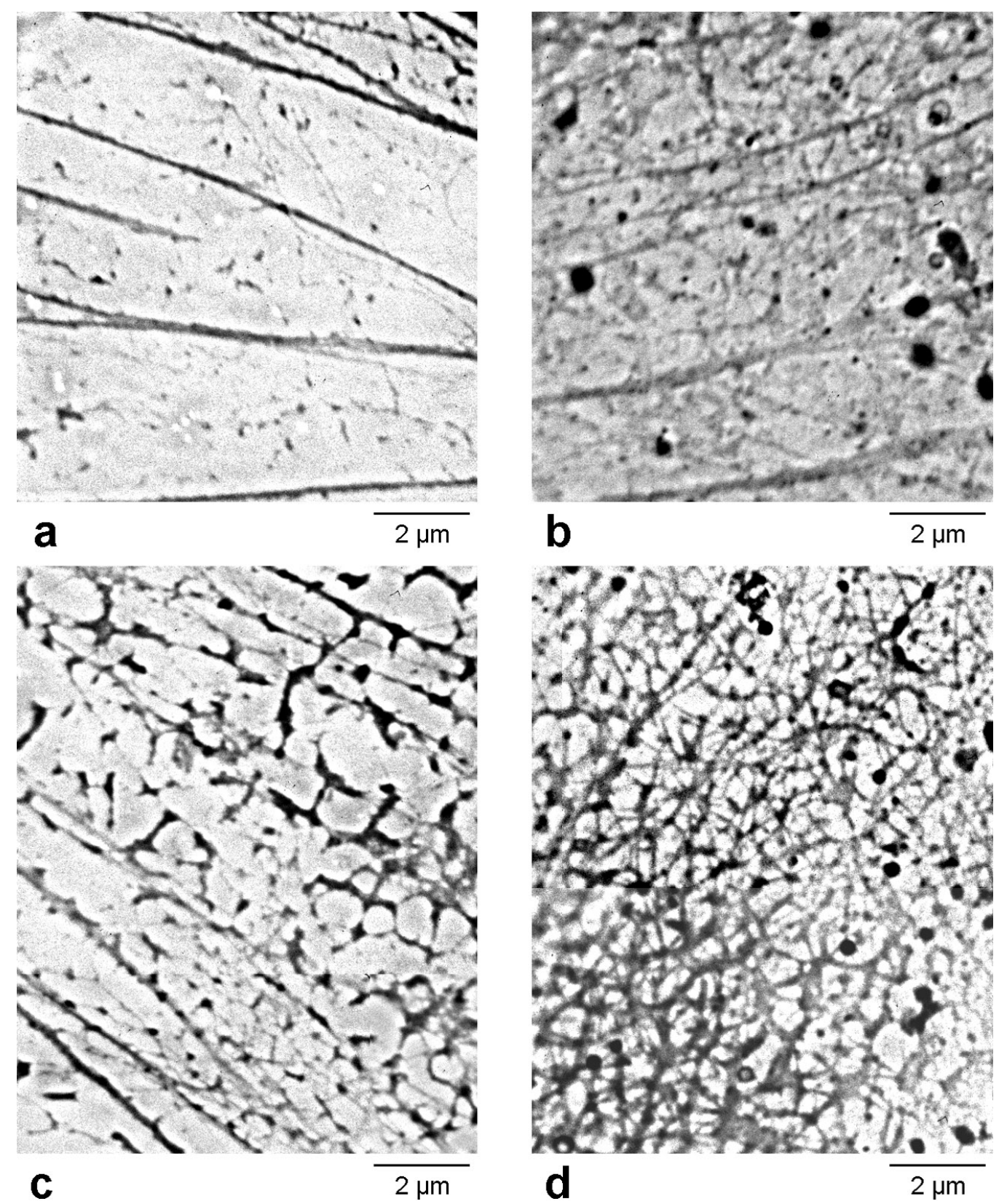

FIG. 4. X-ray micrographs of PtK 2 cytoskeletons comparing the results of varying the times of extraction and of observing the cytoskel etons in either the dry or wet state. (a) Extracted for $1 \mathrm{~min}$, air-dried. (b) Extracted for $1 \mathrm{~min}$, observed as a wet probe. (c and d) As in (a) and (b) but extracted for $3 \mathrm{~min}$. (a) and (c) were stained with uranyl acetate prior to observation in the dry state. Note the better contrast seen in the air-dried probe (a) versus the wet probe (b). Note that the detergent extraction allows stress fibers and other cytoskel etal components to be visualized in the wet probes, whereas without extraction (cf. Fig. 2) thesestructures were not seen. Notethat the longer extraction in (d) gives better contrast than in (b) for the wet probes.

contrast, but also to the Brownian motion of the structures in the wet specimens, which occurs because of the relatively long exposure times required in the phase-contrast mode.

The results in Figs. 2-4 stress that visualization of the cytoskel etal el ements by TXM depends on extracting soluble proteins. In unextracted whol e cells there is not sufficient contrast between the soluble pro- teins in the cytoplasm and the cytoskeletal filaments. After extraction of the soluble proteins, the cytoskeletal filaments, which are insoluble, can be visualized in the X-ray microscope because they now possess sufficient contrast when viewed against a less dense background of extracted cytoplasm. Staining with uranyl acetate is of less importance as can be seen by comparing the contrast seen with the wet 
specimens in Figs. $4 b$ and $4 d$, which have not been stained, with those in Figs. 4a and 4c, which have been stained with uranyl acetate.

Figures 5 and 6 are overviews of two different PtK 2 cells that have been extracted with detergent, air-dried, labeled with uranyl acetate, and observed in the air-dried state. Both cells are characterized by nuclei with a mottled appearance with alternating dark and light areas, which we think is an artifact caused by air-drying of the specimens. In the cell in Fig. 6 the nucleolus can be clearly seen. Elements of the endoplasmic reticulum are visible to the right of thenucleus in Fig. 5, and surrounding the nucleus in Fig. 6. This distribution of endoplasmic reticulum elements parallels that seen by staining with lipidbinding dyes (cf. Terasaki et al., 1984). Thick tightly

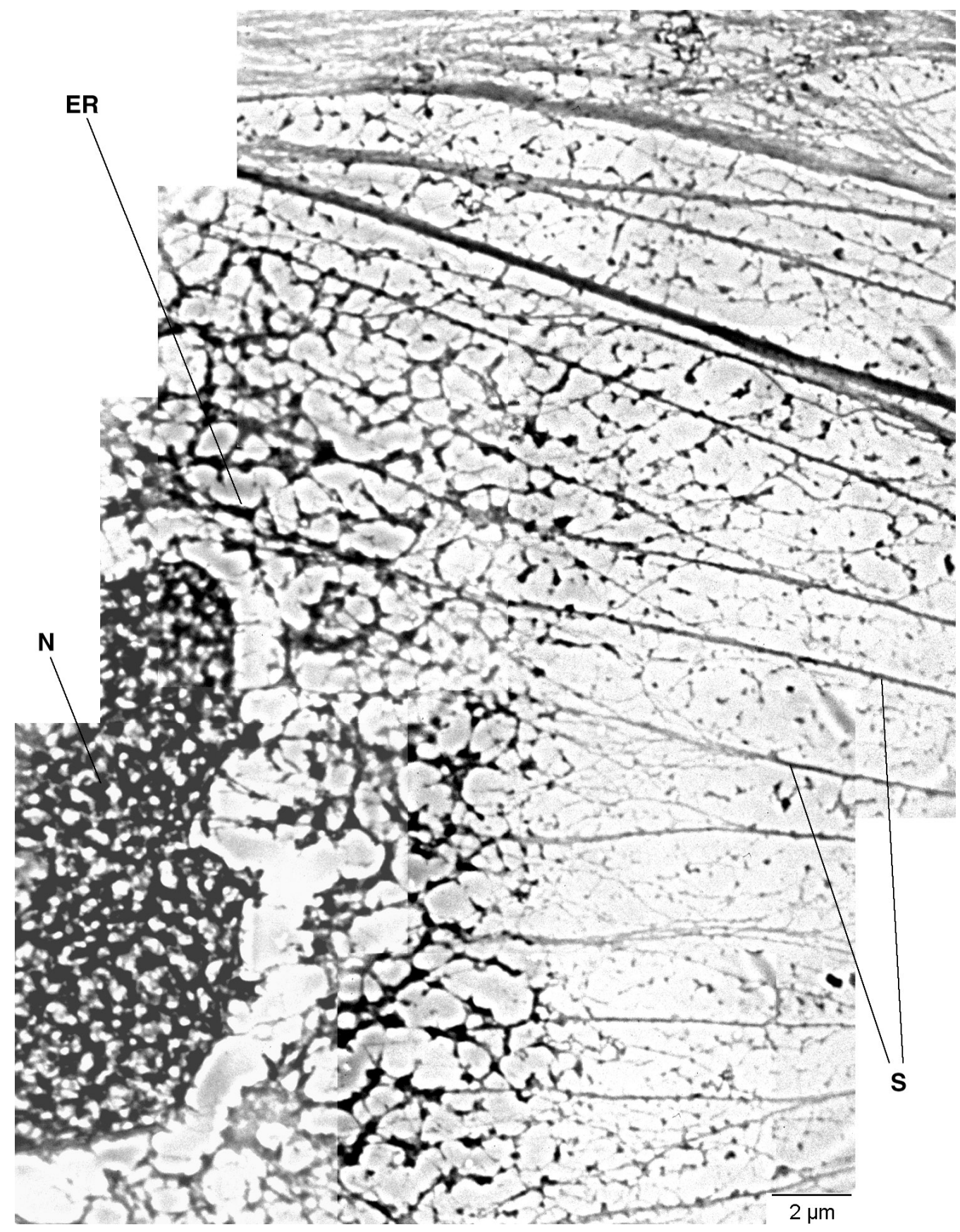

FIG. 5. X-ray micrograph of part of a PtK 2 cell extracted with detergent, air-dried, labeled with uranyl acetate, and observed in the air-dried state. The nucleus (N) surrounded by components of the endoplasmic reticulum (ER) is seen on the left, and stress fibers (S) as well as thinner filaments are visible in the cytoplasm. 
bundled stress fibers are visible in both figures. The individual actin fibers that constitute the stress fibers often appear more spread out at the edge of the cell (e.g., at the top of the cell in Fig. 5). Thinner filaments are visible in the areas between the stress fibers. These have diameters of around $50 \mathrm{~nm}$, as can be calculated from the higher-power view of such filaments in Fig. 7.

Finally it is possible to compare the cytoskeleton visualized by TXM with that seen by TEM. Figure 8 is a transmission electron micrograph of a whole mount preparation of a PtK 2 cytoskeleton prepared by detergent extraction. In the cytoplasm the endoplasmic reticulum is clearly visualized, as are stress fibers and thinner filaments. In the transmission electron microscope the smallest filaments have diameters of around $10 \mathrm{~nm}$. Thus the individual actin filaments (expected diameter, 6-10 nm) and

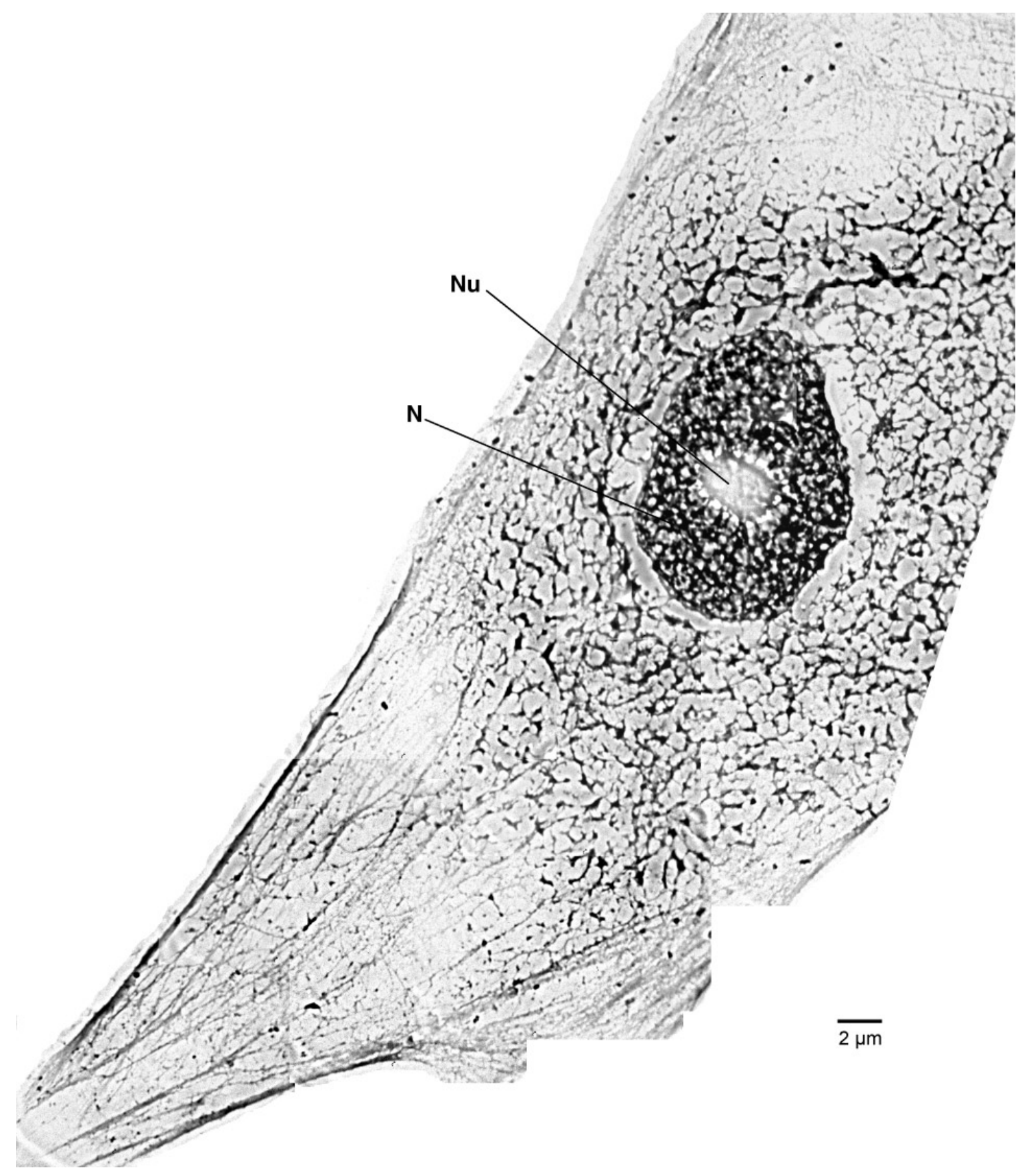

FIG. 6. X-ray micrograph of PtK 2 cytoskel eton prepared as in Fig. 5. Notethe endoplasmic reticulum and the stress fibers visible in the cytoskeleton, as well as the "mottled" appearance of the nucleus (N) and the clearly distinguishable nucleolus (Nu). 


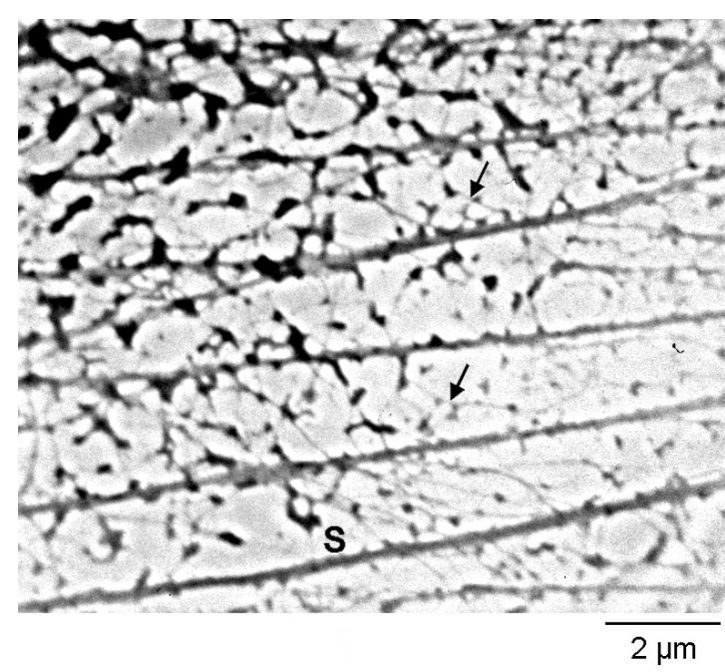

FIG. 7. X-ray micrograph of part of PtK 2 cytoskeleton showing the thinner filaments present between the stress fibers (S). These filaments have a measured diameter of around $50 \mathrm{~nm}$ (see text). The specimen was prepared as in Fig. 5.

the intermediate filaments (expected diameter, 7-11 $\mathrm{nm}$ ) are visualized closer to their real diameters by TEM (cf. Osborn et al., 1978; Webster et al., 1978) than by TXM. No details can be seen in the nuclei in the transmission el ectron microscope.

\section{DISCUSSION}

\section{Visual ization of Cytoskel etons by TXM}

The results reported here demonstrate that the different cytoskeletal elements in interphase PtK2 cells can be visualized by TXM both in the wet state and in air-dried specimens. Thus intracellular fibrous elements such as stress fibers, actin filaments, and intermediate filaments can be clearly visualized for the first time by TXM, and their distributions correspond to those seen earlier by TEM.

The key step in visualizing filaments was to extract the cell with a buffer containing the detergent Triton X-100, a procedure that is known to make holes in the plasma membrane and to extract certain organelles and soluble proteins from the cells. Examination of extracted cells (e.g., Figs. 3-7) reveals long straight stress fibers running through the cytoplasm with a diameter of $120-200 \mathrm{~nm}$, as well as many thinner filaments, with diameters of $\sim 50 \mathrm{~nm}$, which correspond to actin filaments and to intermediate filaments. Microtubules are not seen in these specimens because the buffer used for detergent extraction does not preserve the microtubular array known to be present in whole cells (cf. Osborn and Weber, 1977b). Nuclei as well as elements of the endoplasmic reticulum are al so retained in the specimens.

The X-ray micrographs of the cytoskeleton (Figs. 3-7) can be compared and contrasted with the X-ray micrographs of whole PtK2 cells (Fig. 2). In the cytoskeleton, filamentous structures and the endoplasmic reticulum predominate. In contrast in the whole cell, X-ray microscopy reveals lipid-containing structures including vesicles and mitochondria, but does not show the fibrous elements of the cytoskeleton. These mi crographs demonstrate that theextraction step is necessary, and suggest that the soluble proteins have to be removed so that the Tritoninsoluble cytoskeletal fibers can be visualized because they have a higher carbon content than does the residual cytoplasm. A similar phenomenon was noted in thin-section TEM of epithelial cells in the snail esophagus, where the characteristic morphology of intermediate filaments was seen in the electron micrographs only after the soluble proteins were removed by detergent extraction (Bartnik et al., 1986).

The images of interphase nuclei obtained by TXM in the wet state or after cryofixation are intriguing because some substructure appears to be visible within the nucleus. Figure $2 a$ is a phase-contrast image of an interphase nucleus of an unextracted, unstained PtK 2 cell fixed in glutaral dehyde, and Fig. $2 \mathrm{~b}$ is an image made in the amplitude-contrast mode of an interphase nucleus of an unextracted PtK 2 cell fixed by plunging into liquid ethane. These images can be directly compared with the micrograph of an interphase nucleus of cells handled as in Fig. 2a but imaged in the amplitude-contrast mode (Methe et al., 1997). These images suggest that use of X-ray microscopy, perhaps in conjunction with gold immunolabeling, might reveal additional information about the arrangement and density of nuclear components. Such information could be helpful in deciding between the current very different models of nuclear structure. While some models envision the nucleus as filled with filamentous structures that form a nuclear scaffold (e.g. Fey et al., 1984), more recent models envisage a more dynamic structure (cf. Lamond and Earnshaw, 1998; Gueth-Hallonet et al., 1998).

\section{Comparison of PtK2 Cytoskel etons by TXM and TEM}

A direct comparison is also possible between PtK2 cytoskeletons viewed by TEM (Fig. 8) and TXM (Figs. 5-7). Clearly, very similar overall impressions are obtained by the two techniques showing that, at least for low-power views of the cytoskeleton, X-ray microscopes can provide the same information as seen in the electron microscope. However, there appear also to be some differences. One difference lies in the diameters that are measured for the thinner filaments in the two techniques. Whereas the thinnest filaments we measured in the transmission electron microscope are around $10 \mathrm{~nm}$, in the transmission $\mathrm{X}$-ray microscope the thinnest fila- 


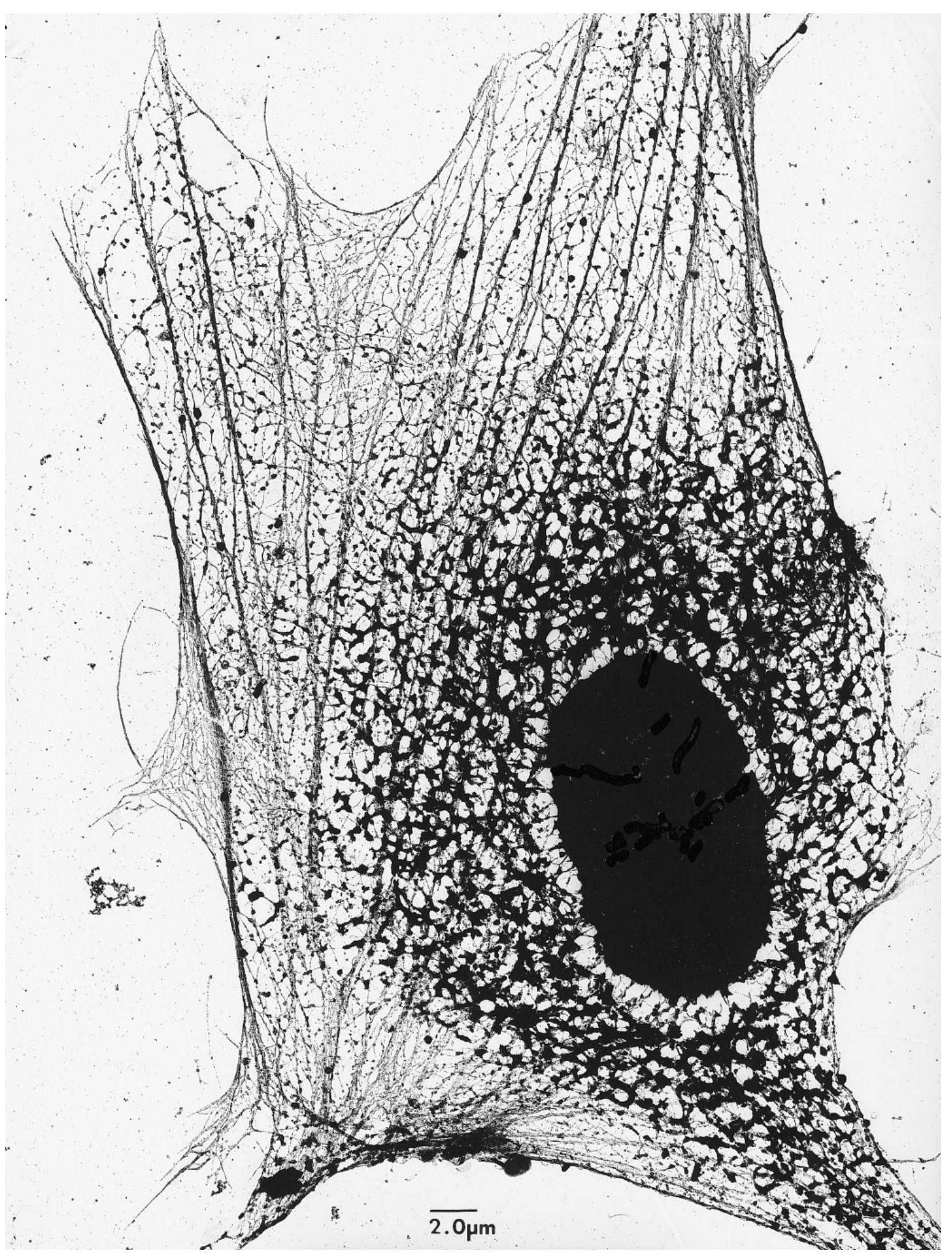

FIG. 8. Whole-mount transmission el ectron micrograph of PtK 2 cytoskeleton. The cell was extracted as in Fig. 5. N ote the similarity to the X-ray micrographs shown in Fig. 5 and 6. A few cracks in the nucleus of this cell have been artifically blackened for aesthetic reasons.

ments have a larger diameter of around $50 \mathrm{~nm}$. This value reflects the limit of resolution for the X-ray microscope which using current optics is around 48 $\mathrm{nm}$. Thus, in the transmission electron microscope actin filaments and intermediate filaments are visu- alized close to their real diameters, whereas in the X-ray microscope they are visualized with a larger diameter, which is equal to the limit of resolution of the transmission X-ray microscope. A second difference appears to be in the structure of the nuclei. In 
the transmission electron microscope, as is well known, no detail is seen because the unsectioned nuclei present in the whole mounts are too thick (cf. Fig. 8). In contrast, in the transmission X-ray microscope, substructure is apparent both in glutaraldehyde-fixed wet specimens (Methe et al., 1997) (Fig. 2e) and, particularly clearly, in the cryofixed cell (Fig. 2b).

\section{Outlook for TXM}

The limit of resolution that can be achieved in the $X$-ray microscope is not, as in the light microscope, limited by the wavelength, but rather by the diffractive X-ray optics (Schmahl et al., 1996). Improvement of the nanostructuring of the zone plate optics should all ow the limit of resolution to be increased to 10-20 nm in the next few years (Schneider et al., 1995b; Weiss et al., 1998). I n addition, improvements in condensers and in X-ray sources should allow a significant reduction in exposure times from the 20-40 s that is currently neccessary for phasecontrast imaging. This should result both in improvements in definition of the objects and in a reduction of the effects of Brownian motion of cellular structures in liquid media occurring while the micrographs are being taken. The X-ray micrographs of whole cytoskel etons shown here (e.g., Figs. 5 and 6) have currently to be assembled from some 30 individual exposures. Use of the new BESSY II el ectron storage ring, rather than BESSY, as well as new condenser arrangements should allow a single cell to be recorded on a single micrograph. This would improve the ease with which X-ray micrographs can be made.

X-ray microscopy at a wavelength of $2.4 \mathrm{~nm}$ detects primarily carbon in biological specimens since water is nearly an order of magnitude moretransparent than organic compounds. The X-ray microscope can be used to provide the density distribution of carbon within the cell in the amplitude-contrast mode. Whole unextracted cells in liquid media show primarily membrane-containing structures such as plasma membranes, nuclear membranes, endoplasmic reticulum components, and mitochondria. Thus, these organelles can be studied directly without additional preparation methods because they show a high contrast to the cytoplasm with which they are in contact. One goal of X-ray microscopy is therefore to examine cells in a state as close to the living state as possible. Radiation damage caused by the $X$ rays does not allow this to be done on living cells. Quickfrozen specimens provide a suitable alternative that approaches the living state and have the further advantage that they can be observed without chemical fixation and are stable to higher X-ray doses. Quick-frozen specimens are comparatively insensi- tive to X-ray damage, and doses up to 10000 that used for living cells can be used without obvious structural alterations in the cells (Schneider et al., 1995a). The cryotechniques can also be used as the basis for a three-dimensional visualization of cells with the help of X-ray tomography as al ready shown for air-dried specimens (Lehr, 1997). In this manner the three-dimensional distribution of carbon density can be documented. A further use of cryotechnology may lie in the investigation of kinetic processes. F or example, specimens can be frozen within $1 \mathrm{~ms}$ so that it is possible to investigate how certain features in the cells change with time (Steinbrecht and Zierold, 1987). In addition, it should be possible to visualize the distribution of specific proteins within the cell using gold-labeled antibodies and TXM, as has already been done in the electron and light microscopes (Griffiths, 1993; De Mey, 1983). Indeed, Chapman et al. (1996) have shown that gold-labeled antibodies can be seen in the scanning transmission $X$-ray microscope in the dark field mode and have used such antibodies to demonstrate the microtubular arrangements in human foreskin fibroblasts in air-dried specimens. However, in the scanning transmission X-ray microscope in the dark field only the gold-labeled antibodies, but not the structure they decorate, has been visualized. Finally, it may be possible, by varying the wavel ength, to obtain distributions of additional elements such as phosphorus and sulfur in the cell. For this purpose, two different imaging modes can be used. It can be done by detection of the element-specific X-ray fluorescence or by transmission images on both sides of the element-specific absorption edges. The biologically relevant concentrations of these elements determine the necessary photon flux for object illumination and therefore the detection limit.

\section{CONCLUSION}

X-ray microscopy allows visualization of the spatial distribution of elements in whole cells or in cytoskeletons in a hydrated condition. At the wavelength of $2.4 \mathrm{~nm}$ used here, primarily carbon is visualized. This results in the visualization of membranes in whole intact cells and, after soluble proteins have been extracted, of cytoplasmic filamentous structures in the cytoskeleton. In the future it may be possible to increase the limit of resolution of the transmission X-ray microscope to $10-20 \mathrm{~nm}$. Clearly the electron microscope has a very much higher resolution, but this is achievable only with samples that are less than $0.1 \mu \mathrm{m}$ thick. In contrast, the X-ray microscope, when used at a wavelength of $2.4 \mathrm{~nm}$, allows substructure to be detected in specimens up to $10 \mu \mathrm{m}$ thick. The fact that under certain conditions substructures can be visualized within 
the cell nucleus further suggests that one application of X-ray microscopy may be to study the cell nucleus, which cannot be done in whole mounts using conventional electron microscopy.

We thank G. Schmahl and D. Rudolph for encouragement and support, Heinz-J ürgen Dehne and J ochen Herbst for expert technical help, and the staff of the BESSY Storage Ring for the excellent working conditions. This study was supported in part by a grant from the German Ministry of Education and Research (BMBF) under Contract 05644 MGA and in part by the Max Planck Society.

\section{REFERENCES}

Bartnik, E., Osborn, M., and Weber, K. (1986) Intermediate filaments in muscle and epithelial cells of nematodes, J. Cell Biol. 102, 2033-2041.

Boomgaard, R. v. d.(1992) Mathematical Morphology: Extensions Towards Computer Vision, Ph.D. thesis, University of Amsterdam.

Chapman, H. N., J acobsen, C., and Williams, S. (1996) A characterisation of dark-field imaging of colloidal gold labels in a scanning transmission x-ray microscope, Ultramicroscopy 62, 191-213.

De Mey, J . (1983) A critical review of light and electron microscopic immunocytochemical techniques used in neurobiology, J . Neurosci. Methods 71, 1-18.

Fey, E. G., Wan, K. M., and Penman, S. (1984) Epithelial cytoskeletal framework and nuclear matrix-intermediate filament scaffold: Three-dimensional organization and protein composition, J . Cell Biol. 98, 1973-1984.

Gilbert, J . R., Pine, J ., Kirz, J ., J acobsen, C., Williams, S., Buckley, C. J ., and Rarback, H. (1992) Soft X-ray absorption imaging of whole wet tissue culture cells, in Michette, A. G., Morisson, G. R., and Buckley, C. J. (Eds.), X-ray Microscopy III, pp. 388-391, Springer, Berlin.

Griffiths, G. (1993) Fine Structure Immunocytochemistry, Springer, Berlin.

Gueth-Hallonet, C., Wang, J., Harborth, J., Weber, K., and Osborn, M. (1998) Induction of a regular nuclear lattice by overexpression of NuMA, Exp. Cell Res. in press.

Kirz, J ., J acobsen, C., and Howells, M. (1995) Soft x-ray microscopes and their biological applications, Q. Rev. Biophys. 28, 33-130.

Lamond, A. I., and Earnshaw, W. C. (1998) Structure and function in the nucleus, Science 280, 547-553.

Lehr, J . (1993) Untersuchungen zum Einsatz von Methoden der digitalen Bildverarbeitung zur Bildaufbereitung in der Röntgenmikroskopie, Diploma thesis, University of Göttingen.

Lehr, J . (1997) 3D X-ray microscopy: Tomographic imaging of mineral sheaths of bacteria Leptothrix Ochracea with the Göttingen x-ray microscope at BESSY, Optik 104, 166-170.

Lenk, R., Ransom, L., Kaufmann, Y., and Penman, S. (1977) A cytoskeletal structure with associated polyribosomes obtained from Hela cells, Cell 10,67-78.

Methe, O., Spring, H., Guttmann, P., Schneider, G., Rudolph, D., Trendelenburg, M. F., and Schmahl, G. (1997) Transmission x-ray microscopy of intact hydrated PtK 2 cells during the cell cycle, J . Microsc. 188, 125-135.

Niemann, B., Schneider, G., Guttmann, P., Rudolph, D., and Schmahl, G. (1994) The new Göttingen X-ray microscope with object holder in air for wet specimen, in X-Ray Microscopy IV, pp. 66-75.

Osborn, M., and Weber, K. (1977a) The detergent-resistant cytoskeleton of tissue culture cells includes the nucleus and the microfilament bundles, Exp. Cell Res. 106, 339-349.

Osborn, M., and Weber, K. (1977b) The display of microtubules in transformed cells, Cell 12, 561-571.

Osborn, M., Webster, R. E., and Weber, K. (1978) Individual microtubules viewed by immunofluorescence and electron microscopy in the same PtK2 cell, J . Cell Biol. 77, R27-R34.

Schmahl, G., Rudolph, D., Niemann, B., and Christ, O. (1980) Zone-plate X-ray microscopy, Q. Rev. Biophys. 13, 297-315.

Schmahl, G., Rudolph, D., Guttmann, P., Schneider, G., Thieme, J ., and Niemann, B. (1995) Phase contrast studies of biological specimens with the x-ray microscope at BESSY, Rev. Sci. Instrum. 66, 1282-1286.

Schmahl, G., Rudol ph, D., Schneider, G., Thieme, J ., Kaulich, B., and Hettwer, M. (1996) Diffraction optics for X-ray imaging, Microel ectronic Eng. 32, 351-357.

Schneider, G. (1992) Röntgenmikroskopie mit Synchrotronstrahlung an wässrigen biologischen Systemen-experimentelle und theoretische Untersuchungen, Thesis, University of Göttingen.

Schneider, G., Niemann, B., Guttmann, P., Rudolph, D., and Schmahl, G. (1995a) Cryo X-ray microscopy, Synchrotron Radiat. News 8, 19-28.

Schneider, G., Schliebe, T., and Aschoff, H. (1995b) Cross-linked polymers for nanofabrication of high resolution zone plates in nickel and germanium, J . Vac. Sci. Technol. 13, 2809-2812.

Steinbrecht, R. A., and Zierold, K. (1987) Cryotechniques in Biological Electron Microscopy, Springer Verlag, Berlin.

Terasaki, M., Song, J ., Wong, J . R., Weiss, M. J ., and Chen, L. B. (1984) Localization of endoplasmic reticulum in living and glutaraldehyde fixed cells with fluorescent dyes, Cell 38, 101108.

Weber, K., Rathke, P. C., and Osborn, M. (1978) Cytoplasmic microtubular images in glutaraldehyde-fixed tissue culture cells by electron microscopy and by immunofluorescence microscopy, Proc. Natl. Acad. Sci. USA 75, 1820-1824.

Webster, R. E., Osborn, M., and Weber, K. (1978) Visualization of the same PtK 2 cytoskeletons by both immunofluorescence and low power electron microscopy, Exp. Cell Res. 117, 47-61.

Weiss, D., Peuker, M., and Schneider, G. (1998) Radiationenhanced network formation in copolymer galvanoforms for diffractive nickel X-ray optics with high aspect ratios, Appl. Phys. Lett. 72, 1805-1807.

Wolter, H. (1952) Spiegelsysteme streifenden Einfalls als abbildende Optiken für Röntgenstrahlen, Ann. Phys. 10, 94-114. 